\title{
Ekeland's variational principle in complete quasi-G-metric spaces
}

\author{
E. Hashemi ${ }^{\mathrm{a}}$, M. B. Ghaemi ${ }^{\mathrm{b}, *}$ \\ ${ }^{a}$ Department of Mathematics, College of Basic Sciences, Karaj Branch, Islamic Azad University, Alborz, Iran. \\ ${ }^{b}$ Department of Mathematics, Iran University of Science and Technology, Tehran, Iran.
}

\begin{abstract}
In this paper, by concept of $\Gamma$-function which is define on $q-G-m$ (quasi-G-metric) space, we establish a generalized Ekeland's variational principle in the setting of lower semicontinuous from above. As application we prove generalized flower petal theorem in q-G-m.
\end{abstract}

Keywords: $\Gamma$-Function, q-G-m space, generalized EVP, lower semicontinuous from above function, generalized Caristi's (common) fixed point theorem, nonconvex minimax theorem, generalized flower petal theorem.

2010 MSC: 54H25, 54C60.

(C)2019 All rights reserved.

\section{Introduction}

EVP was first studied in 1972. Many equivalents have been found by scholars over the years for primitive EVP[10, 11], see [2-7, 17, 18, 20, 22]. Interesting applications in various fields of applied mathematics are found. A number of generalized of these results have been reviewed by other researchers $[1-4,8,12-16,23-30]$.

\section{Ekeland's variational principle}

In this paper, $\theta:(-\infty, \infty) \longrightarrow(0, \infty)$ is a nondecreasing function, a function $\mathrm{g}: \mathrm{U} \longrightarrow(-\infty, \infty)$ is said to be lower semicontinuous from above (shortly Lsca) at $r_{0}$, when for each sequence $\left\{r_{n}\right\}$ in $U$ such that $r_{n} \longrightarrow r_{0}$ and $g\left(r_{1}\right) \geqslant g\left(r_{2}\right) \geqslant \cdots \geqslant g\left(r_{n}\right) \geqslant \cdots$, we have $g\left(r_{0}\right) \leqslant \lim _{n \rightarrow \infty} g\left(r_{n}\right)$. The function $g$ is said to be Lsca on $\mathrm{U}$, when $\mathrm{g}$ is Lsca at every point of $\mathrm{U}, \mathrm{g}$ is proper when $\mathrm{h} \not \equiv \infty$.

Theorem 2.1 ([9, Ekeland theorem]). Let $\mathrm{U}$ be a complete metric space with meter $\mathrm{d}, \mathrm{g}: \mathrm{U} \longrightarrow \mathbb{R} \cup\{+\infty\}$ be a proper, semicontinuous, and bounded below function. Then there exists $v \in \mathrm{U}$ such that $\mathrm{g}(v) \leqslant \mathrm{g}(\mathrm{u}), \mathrm{d}(\mathrm{u}, v) \leqslant 1$, and $\mathrm{g}(w)>\mathrm{g}(v)-\epsilon \mathrm{d}(v, w)$ for all $v \neq w$.

\footnotetext{
*Corresponding author

Email addresses: eshagh_hashemi@yahoo.com (E. Hashemi), mghaemi@iust.ac.ir (M. B. Ghaemi)

doi: $10.22436 /$ jnsa.012.03.06
}

Received: 2018-01-19 Revised: 2018-09-28 Accepted: 2018-10-25 
Definition 2.2 ([19]). Assume that $\mathrm{U}$ is a nonempty set and mapping

$$
\mathrm{G}: \mathrm{U} \times \mathrm{U} \times \mathrm{U} \longrightarrow[0, \infty)
$$

is satisfying the following conditions:

(i) $\mathrm{G}(\mathrm{r}, \mathrm{s}, \mathrm{t})=0$ if $\mathrm{r}=\mathrm{s}=\mathrm{t}$;

(ii) $G(r, r, s)>0$ for all $r, s \in U$, where $r \neq s$;

(iii) $G(r, r, t) \leqslant G(r, s, t)$ for all $r, s, t \in U$ with $r \neq t$;

(iv) $G(r, s, t)=G(p\{r, s, t\})$ such that $p$ is a permutation of $r, s, t$;

(v) $G(r, s, t) \leqslant G(r, \alpha, \alpha)+G(\alpha, s, t)$ for all $r, s, t, \alpha$ in $U$.

Then $\mathrm{G}$ is said to be $\mathrm{G}$-metric and pair $(\mathrm{U}, \mathrm{G})$ is said to be $\mathrm{G}$-metric space.

Definition 2.3 ([19]). Let $(\mathrm{U}, \mathrm{G})$ be a G-metric space. A sequence $\left\{r_{n}\right\}$ in $U$ is said to be

(a) G-Cauchy sequence if for all $\epsilon>0$, there exists $q_{0} \in \mathbb{N}$ such that for every $p, q, l \in \mathbb{N}$ and $p, q, l \geqslant q_{0}$ then $G\left(r_{q}, r_{m}, r_{l}\right)<\epsilon$;

(b) G-convergent to $r \in U$ if for all $\epsilon>0$, there exists natural number $q_{0}$ such that for all $p, q \geqslant q_{0}$, then $\mathrm{G}\left(\mathrm{r}_{\mathrm{q}}, \mathrm{r}_{\mathrm{p}}, \mathrm{r}\right)<\epsilon$.

Proposition 2.4 ([19]). Assume that $(\mathrm{U}, \mathrm{G})$ is a $\mathrm{G}$-metric space, then the following statements are equivalent:

(a) $\left\{r_{n}\right\}$ is a G-caushy sequence;

(b) for each $\epsilon>0$, there exists natural number $\mathrm{q}_{0}$ such that for all $\mathrm{p}, \mathrm{q} \geqslant \mathrm{q}_{0}$, then $\mathrm{G}\left(\mathrm{r}_{\mathrm{q}}, \mathrm{r}_{\mathrm{p}}, \mathrm{r}_{\mathrm{p}}\right)<\epsilon$.

Definition 2.5. A function $\sigma: \mathbb{R}_{+} \longrightarrow \mathbb{R}_{+}$is subaddtive when $\sigma(r+s) \leqslant \sigma(r)+\sigma(s)$, and $\sigma(\epsilon r)=\epsilon \sigma(r)$ for every $\epsilon>0$.

Definition 2.6. Let $\mathrm{U}$ be a nonempty set. A function

$$
\mathrm{G}: \mathrm{U} \times \mathrm{U} \times \mathrm{U} \longrightarrow[0, \infty)
$$

is said to be quasi-G-metric (q-G-m) if the following conditions be satisfied

1. $G(r, s, t)=0$ if $r=s=t$;

2. $G(r, r, s)>0$ for all $r, s \in U, r \neq s$;

3. $G(r, r, t) \leqslant G(r, s, t)$ for all $r, s, t \in U, t \neq s$;

4. $G(r, s, t) \leqslant G(r, \varepsilon, \varepsilon)+G(\varepsilon, s, t)$ for all $r, s, t, \varepsilon \in U$.

$(\mathrm{U}, \mathrm{G})$ is said to be $\mathrm{q}-\mathrm{G}-\mathrm{m}$ space when $\mathrm{U}$ is a nonempty set and $\mathrm{G}$ is a $\mathrm{q}-\mathrm{G}-\mathrm{m}$. The concept of Cauchy sequence, convergence, and complete space are defined as G-metric space.

Definition 2.7. Let $(\mathrm{U}, \mathrm{G})$ be a $\mathrm{q}-\mathrm{G}$-m space. A function $\Gamma: \mathrm{U} \times \mathrm{U} \times \mathrm{U} \longrightarrow[0, \infty)$ is said to be $\Gamma$-function when

(1) $\Gamma(r, s, t) \leqslant \Gamma(r, \varepsilon, \varepsilon)+\Gamma(\varepsilon, s, t)$ for all $r, s, t, \varepsilon \in U$;

(2) if $r \in U,\left\{s_{n}\right\}_{n \in \mathbb{N}}$ be a sequence in $U$ which is convergent to $s$ in $U$ and $\Gamma\left(r, s_{n}, s_{n}\right) \leqslant M$, then $\Gamma(r, s, s) \leqslant M$

(3) for every $\epsilon>0$, there exists $\delta>0$ such that $\Gamma(r, \varepsilon, \varepsilon) \leqslant \delta$ and $\Gamma(\varepsilon, s, t) \leqslant \delta$ imply $G(r, s, t) \leqslant \epsilon$.

Example $2.8([21])$. Let $(U, d)$ be a metric space and $G: U^{3} \longrightarrow[0, \infty)$ defined by $G(r, s, t)=\max \{d(r, s)$, $d(r, t), d(s, t)\}$ for all $r, s, t \in U$. Then $\Gamma=G$ is a $\Gamma$-function on $U$.

Example 2.9. Assume that

$$
\mathrm{G}: \mathrm{U}^{3} \longrightarrow[0, \infty), \quad \mathrm{G}(\mathrm{r}, \mathrm{s}, \mathrm{t})=\frac{1}{3}(|\mathrm{t}-\mathrm{r}|+|\mathrm{r}-\mathrm{s}|)
$$

is a function, then $\mathrm{G}$ is a q-G-m but isn't $\mathrm{G}$ metric. 
Proof. $\mathrm{q}-\mathrm{G}-\mathrm{m}$ is obvious. We show that $\mathrm{G}(\mathrm{r}, \mathrm{s}, \mathrm{t}) \neq \mathrm{G}\{\mathrm{p}(\mathrm{r}, \mathrm{s}, \mathrm{t})\}$ (p is a permutation of $r, \mathrm{~s}, \mathrm{t}$ ). Since

$$
\mathrm{G}(3,5,2)=\frac{1}{3}(|2-3|+|3-5|)=1, \quad \mathrm{G}(2,3,5)=\frac{1}{3}(|3-2|+|5-2|)=\frac{4}{3},
$$

then $\mathrm{G}$ is not a $\mathrm{G}$-metric.

Example 2.10. Let $G(r, s, t)$ be the same as in the previous example. Then $\Gamma=G$ is a $\Gamma$-function.

Proof. (a) and (b) are obvious. Let $\epsilon>0$ be given, put $\delta=\frac{\epsilon}{2}$ if $\Gamma(r, \varepsilon, \varepsilon)=\frac{1}{3}(|t-\varepsilon|+|\varepsilon-s|)<\frac{\epsilon}{2}$, then

$$
\mathrm{G}(\mathrm{r}, \mathrm{s}, \mathrm{t})=\frac{1}{3}(|\mathrm{t}-\mathrm{s}|+|\mathrm{r}-\mathrm{s}|) \leqslant \frac{1}{3}(|\mathrm{t}-\varepsilon|+|\varepsilon-\mathrm{r}|+|\mathrm{r}-\varepsilon|+|\varepsilon-\mathrm{s}|)<\epsilon .
$$

So, (c) is established.

Lemma 2.11 ([21]). Assume that $(\mathrm{U}, \mathrm{G})$ is a G-metric space and $\Gamma$ is a $\Gamma$-function on $\mathrm{U}$. Let $\left\{\mathrm{u}_{\mathrm{n}}\right\}$ and $\left\{v_{\mathrm{n}}\right\}$ be two sequences in $\mathrm{U},\left\{\rho_{\mathrm{n}}\right\}$ and $\left\{\phi_{\mathrm{n}}\right\}$ be in $[0, \infty]$, which are convergent to zero. Let $\mathrm{u}, v, w, \varepsilon \in \mathrm{U}$, then

(1) if $\Gamma\left(v, u_{n}, u_{n}\right) \leqslant \rho_{n}$ and $\Gamma\left(u_{n}, v, w\right) \leqslant \phi_{n}$ for all $n \in \mathbb{N}$, then $G(v, v, w)<\epsilon$ and hence $w=v$;

(2) if $\Gamma\left(u_{n}, u_{n}, u_{n}\right) \leqslant \rho_{n}$ and $\Gamma\left(u_{n}, u_{m}, w\right) \leqslant \phi_{n}$ for every $m>n$, then $G\left(v_{n}, v_{m}, w\right)$ is convergent to zero and hence $v_{\mathrm{n}} \rightarrow w$;

(3) if $\Gamma\left(u_{n}, u_{m}, u_{l}\right) \leqslant \rho_{n}$ for all $m, n, l \in \mathbb{N}$ with $n \leqslant m \leqslant l$, then $\left\{u_{n}\right\}$ is a G-Cauchy sequence;

(4) if $\Gamma\left(u_{n}, \varepsilon, \varepsilon\right) \leqslant \rho_{n}$ for all $n \in \mathbb{N}$, then $\left\{u_{n}\right\}$ is a G-Cauchy sequence.

Lemma 2.12. Let $\Gamma$ be a $\Gamma$-function on $\mathrm{U} \times \mathrm{U} \times \mathrm{U}$. If sequence $\left\{r_{n}\right\}$ be in $\mathrm{U}$ that $\lim \sup \left\{\Gamma\left(\mathrm{r}_{\mathrm{n}}, \mathrm{r}_{\mathrm{m}}, \mathrm{r}_{\mathrm{l}}\right), \mathrm{n} \leqslant \mathrm{m} \leqslant\right.$ $l\}=0$, then $\left\{\mathrm{r}_{\mathrm{n}}\right\}$ will be a G-Cauchy sequence in $\mathrm{U}$.

Proof. Assume $\rho_{n}=\sup \left\{\Gamma\left(r_{n}, r_{m}, r_{l}\right)\right\}$, then $\lim _{n \rightarrow \infty} \rho_{n}=0$. By Lemma 2.11 (3), $\left\{r_{n}\right\}$ is a G-Cauchy sequence.

Lemma 2.13. Let $\mathrm{g}: \mathrm{U} \longrightarrow[-\infty, \infty]$ be a function and $\Gamma$ be $a \Gamma$-function on $\mathrm{U} \times \mathrm{U} \times \mathrm{U}$. The set $\mathrm{P}(\mathrm{r})$ is defined by

$$
\mathrm{P}(\mathrm{r})=\{\mathrm{s} \in \mathrm{U} ; \mathrm{s} \neq \mathrm{r}, \quad \Gamma(\mathrm{r}, \mathrm{s}, \mathrm{s}) \leqslant \theta(\mathrm{g}(\mathrm{r}))(\mathrm{g}(\mathrm{r})-\mathrm{g}(\mathrm{s}))\} .
$$

If $\mathrm{P}(\mathrm{r})$ be nonempty, then for every $\mathrm{s} \in \mathrm{P}(\mathrm{r})$, we will have

$$
\mathrm{P}(\mathrm{s}) \subseteq \mathrm{P}(\mathrm{r}) \text { and } \mathrm{g}(\mathrm{s}) \leqslant \mathrm{g}(\mathrm{r}) .
$$

Proof. Let $s \in P(r)$. So $s \neq r$ and $\Gamma(r, s, s) \leqslant \theta(g(r))(g(r)-g(s))$. Since $\Gamma(r, s, s) \geqslant 0$ and $\theta$ is nondecreasing and positive function, then $g(r) \geqslant g(s)$. If $P(s)=\emptyset$ then $P(s) \subseteq P(r)$. Therefore $t \neq s$ and $\Gamma(s, t, t) \leqslant$ $\theta(g(s))(g(s)-g(t))$ as above $g(s) \geqslant g(t)$. Since $\Gamma$ be a $\Gamma$-function, then

$$
\Gamma(r, t, t) \leqslant \Gamma(r, s, s)+\Gamma(s, t, t) \leqslant \theta(g(r))(g(r)-g(t)) .
$$

We claim that $t \neq r$. Assume that $t=r$ so $\Gamma(r, t, t)=0$. On the other hand

$$
\Gamma(r, s, s) \leqslant \theta(g(r))(g(r)-g(s)) \leqslant \theta(g(r))(g(r)-g(t))=0 \Longrightarrow \Gamma(r, s, s)=0,
$$

then $\Gamma(r, s, s)=0$. For every $\epsilon>0$, we have $\Gamma(r, t, t)=0<\epsilon$ and $\Gamma(t, s, s)=0<\epsilon$ then by definition $\Gamma$-function, we have $G(t, s, s)<\epsilon$, so $G(t, s, s)=0$ and $t=s$. This is a contradiction, therefore $t \in P(r)$ and $\mathrm{P}(\mathrm{s}) \subseteq \mathrm{P}(\mathrm{r})$.

Proposition 2.14. Assume that $(\mathrm{U}, \mathrm{G})$ is a complete $\mathrm{q}-\mathrm{G}-\mathrm{m}$ space and $\mathrm{g}: \mathrm{U} \longrightarrow[-\infty, \infty]$ is a proper and bounded below function, $\Gamma$ is a $\Gamma$-function on $\mathrm{U} \times \mathrm{U} \times \mathrm{U}$. Let

$$
\mathrm{P}(\mathrm{r})=\{\mathrm{s} \in \mathrm{U} ; \mathrm{s} \neq \mathrm{r}, \Gamma(\mathrm{r}, \mathrm{s}, \mathrm{s}) \leqslant \theta(\mathrm{g}(\mathrm{r}))(\mathrm{g}(\mathrm{r})-\mathrm{g}(\mathrm{s}))\} .
$$

Let $\left\{r_{n}\right\}$ be a sequence in $\mathrm{U}$ such that $\mathrm{P}\left(\mathrm{r}_{\mathrm{n}}\right)$ be nonempty and for all $\mathrm{n} \in \mathbb{N}, \mathrm{r}_{\mathrm{n}+1} \in \mathrm{P}\left(\mathrm{r}_{\mathrm{n}}\right)$. Then, there exists 
$r_{0} \in U$ such that $r_{n} \longrightarrow r_{0}$ and $r_{0} \in \bigcap_{n=1}^{\infty} P\left(r_{n}\right)$. Also if for every $n \in \mathbb{N}$, we have $g\left(r_{n+1}\right) \leqslant \inf _{t \in P\left(r_{n}\right)} g(t)+\frac{1}{n}$, then $\bigcap_{n=1}^{\infty} \mathrm{P}\left(\mathrm{r}_{\mathrm{n}}\right)$ will only has one member.

Proof. At first we prove that $\left\{r_{n}\right\}$ is a Cauchy sequence by Lemma 2.13, $g\left(r_{n}\right) \geqslant g\left(r_{n+1}\right)$ for all $n \in \mathbb{N}$. Therefore $\left\{g\left(r_{n}\right)\right\}$ is nonincreasing. On the other hand $g$ is bounded below then $\lim _{n \rightarrow \infty} g\left(r_{n}\right)=u$, and $g\left(r_{n}\right) \geqslant u$ for all $n \in \mathbb{N}$. We claim that

$$
\limsup _{n \rightarrow \infty}\left\{\Gamma\left(r_{n}, r_{m}, r_{m}\right): m>n\right\}=0 .
$$

We have

$$
\begin{aligned}
\Gamma\left(r_{n}, r_{m}, r_{m}\right) & \leqslant \Gamma\left(r_{n}, r_{n+1}, r_{n+1}\right)+\Gamma\left(r_{n+1}, r_{m}, r_{m}\right) \\
& \leqslant \Gamma\left(r_{n}, r_{n+1}, r_{n+1}\right)+\Gamma\left(r_{n+1}, r_{n+2}, r_{n+2}\right)+\cdots+\Gamma\left(r_{m-1}, r_{m}, r_{m}\right),
\end{aligned}
$$

then

$$
\Gamma\left(r_{n}, r_{m}, r_{m}\right) \leqslant \sum_{j=n}^{m-1} \Gamma\left(r_{n}, r_{m}, r_{m}\right) \leqslant \theta(g(r))\left(g\left(r_{n}\right)-u\right)
$$

for all $m, n \in \mathbb{N}$ with $m>n$.

Put $\rho_{n}=\theta(g(r))\left(g\left(r_{n}\right)-u\right)$, then $\sup \left\{\Gamma\left(r_{n}, r_{m}, r_{m}\right): m>n\right\} \leqslant \rho_{n}$. for all $n \in \mathbb{N}$. Since $\lim _{n \rightarrow \infty} g\left(r_{n}\right)=$ $u$, we result

$$
\limsup _{n \rightarrow \infty}\left\{\Gamma\left(r_{n}, r_{m}, r_{m}\right): m>n\right\}=0
$$

and $\lim _{n \rightarrow \infty} \rho_{n}=0$. By Lemma 2.12, $\left\{u_{n}\right\}$ is a G-Cauchy sequence. Then, there exists $r_{0} \in U$ such that $r_{n} \rightarrow u_{0}$. We show that $r_{0} \in \bigcap_{n=1}^{\infty} P\left(r_{n}\right)$. Since $g$ is Lsca, then $g\left(r_{0}\right) \leqslant \lim _{n \rightarrow \infty} g\left(r_{n}\right)=u \leqslant g\left(r_{k}\right)$.

Let $n \in \mathbb{N}$, we have

$$
\Gamma\left(r_{n}, r_{m}, r_{m}\right) \leqslant \sum_{j=n}^{m-1} \Gamma\left(r_{j}, r_{j+1}, r_{j+1}\right) \leqslant \theta\left(g\left(r_{n}\right)\right)\left(g\left(r_{n}\right)-g\left(r_{0}\right)\right)
$$

for all $m \in \mathbb{N}$ with $m>n$. By Definition 2.7 (2), we have

$$
\Gamma\left(r_{n}, r_{0}, r_{0}\right) \leqslant \theta\left(g\left(r_{n}\right)\left(g\left(r_{n}\right)-g\left(r_{0}\right)\right)\right.
$$

for all $n \in \mathbb{N}$. Also $r_{0} \neq r$ for all $n \in \mathbb{N}$, suppose it is not, then there exists $j \in \mathbb{N}$ such that $r_{0}=r_{j}$. Since

$$
\Gamma\left(r_{j}, r_{j+1}, r_{j+1}\right) \leqslant \theta\left(g\left(r_{j}\right)\right)\left(g\left(r_{j}\right)-g\left(r_{j+1}\right)\right) \leqslant \theta\left(g\left(r_{j}\right)\right)\left(g\left(r_{j}\right)-g\left(r_{0}\right)\right)=0,
$$

then we have $\Gamma\left(r_{j}, r_{j+1}, r_{j+1}\right)=0$ and in the same way

$$
\Gamma\left(r_{j+1}, r_{j+2}, r_{j+2}\right)=0 .
$$

Now assume $\epsilon>0, \Gamma\left(r_{j}, r_{j+1}, r_{j+1}\right)=0<\delta$, and $\Gamma\left(r_{j+1}, r_{j+2}, r_{j+2}\right)=0<\delta$. Therefor by Definition 2.7 (3) we get it $G\left(r_{j}, r_{j+2}, r_{j+2}\right)<\epsilon$. Then $r_{j}=r_{j+2}$ that is a contradiction because of $r_{j} \neq r_{j+2}$. Since $r_{j+1} \in P\left(r_{j}\right)$, then $P\left(r_{j+1}\right) \subseteq P\left(r_{j}\right)$ and $r_{j+2} \in P\left(r_{j+1}\right)$. So $r_{j+2} \in P\left(r_{j}\right)$. We suppose $r_{j+2} \neq r_{j}$ for all $n \in \mathbb{N}$. We have $r_{0} \in \bigcap_{n=1}^{\infty} P\left(r_{n}\right)$, then $\bigcap_{n=1}^{\infty} P\left(r_{n}\right) \neq \emptyset$. Let $g\left(r_{n+1}\right) \leqslant \inf _{t \in P\left(r_{n}\right)} g(t)+\frac{1}{n}$ for all $r_{0} \neq r_{n}$. We show that $\bigcap_{n=1}^{\infty} P\left(r_{n}\right)=\left\{r_{0}\right\}$. Assume that $w \in \bigcap_{n=1}^{\infty} P\left(r_{n}\right)$, then

$$
\Gamma\left(r_{n}, w, w\right) \leqslant \theta\left(g\left(r_{n}\right)\right)\left(g\left(r_{n}\right)-g(w)\right) \leqslant \theta\left(g\left(r_{1}\right)\right)\left(g\left(r_{n}\right)-\inf _{t \in P\left(r_{n}\right)} g(t)\right) \leqslant \theta\left(g\left(r_{1}\right)\right)\left(g\left(r_{n}\right)-g\left(r_{n+1}\right)+\frac{1}{n}\right) .
$$


Let

$$
\varphi_{\mathrm{n}}=\theta\left(g\left(r_{1}\right)\right)\left(g\left(r_{n}\right)-g\left(r_{n+1}\right)+\frac{1}{n}\right)
$$

for all $n \in \mathbb{N}$, then $\lim _{n \rightarrow \infty} \varphi_{n}=0$, we get it $\lim _{n \rightarrow \infty} \Gamma\left(r_{n}, w, w\right)=0$. On the other hand $\left\{r_{m}\right\}$ is a GCauchy sequence. Then $\lim _{n \rightarrow \infty} \Gamma\left(r_{m}, r_{m}, r_{n}\right)=0$ and we get it $r_{n} \rightarrow \infty$, by uniqueness $w=r_{0}$. Then $\bigcap_{n=1}^{\infty} P\left(r_{n}\right)=\left\{r_{0}\right\}$

Theorem 2.15 (Generalized Ekeland's variational principle). Assume that $(\mathrm{U}, \mathrm{G})$ is a complete $q-\mathrm{G}-m$ space and $\mathrm{g}: \mathrm{U} \longrightarrow(-\infty, \infty]$ be a proper, bounded below and Lsca function. $\Gamma$ is a $\Gamma$-function on $\mathrm{U} \times \mathrm{U} \times \mathrm{U}$, then there exists $\mathrm{r} \in \mathrm{U}$ such that

$$
\Gamma(v, r, r)>\theta(g(r))(g(r)-g(v))
$$

for all $\mathrm{r} \in \mathrm{U}$ with $v \neq \mathrm{r}$.

Proof. Suppose it isn't true. Then for every $r \in U$, there exists $s \in U, s \neq r$ such that $\Gamma(r, s, s) \leqslant$ $\theta(g(r))(g(r)-g(s))$. That is $P(r) \neq \emptyset$. We define the sequence $\left\{r_{n}\right\}$ as follows. Put $r_{1}=\varepsilon$, we choose $r_{2} \in P\left(r_{1}\right)$ such that $g\left(r_{2}\right) \leqslant \inf _{r \in P\left(r_{1}\right)} g(r)+1$. In the same way suppose that $r_{n} \in U$ is given. We choose $r_{n+1} \in P\left(r_{n}\right)$ such that $g\left(r_{n+1}\right) \leqslant \inf _{r \in P\left(r_{n}\right)} g(r)+\frac{1}{n}$. By proposition 2.14, there exists $r_{0} \in U$ such that

$$
\bigcap_{n=1}^{\infty} P\left(r_{n}\right)=\left\{r_{0}\right\} .
$$

By lemma 2.13, we have $P\left(r_{0}\right) \subseteq \bigcap_{n=1}^{\infty} P\left(r_{n}\right)=\left\{r_{0}\right\}$ then $P\left(r_{0}\right)=\left\{r_{0}\right\}$. This is a contradiction. Therefore there exists $v \in \mathrm{U}$ such that

$$
\Gamma(v, r, r)>\theta(g(v))(g(v)-g(r)) .
$$

Theorem 2.16 (Generalized Caristi's common fixed point theorem for a family of multivalued maps). Assume that $(\mathrm{U}, \mathrm{G})$ is a complete $q-\mathrm{G}-\mathrm{m}$ space and $\mathrm{g}: \mathrm{U} \longrightarrow(-\infty, \infty]$ be a proper, bounded below and Lsca function. $\Gamma$ is a $\Gamma$-function on $\mathrm{U} \times \mathrm{U} \times \mathrm{U}$. Let $\mathrm{J}$ be any index set and for each $\mathrm{j} \in \mathrm{J}$, suppose $\mathrm{T}_{\mathrm{j}}: \mathrm{U} \rightarrow 2^{\mathrm{U}}$ is multivalued map such that for each $\mathrm{r} \in \mathrm{U}$, there is $s=s(r, j) \in \mathrm{T}_{\mathbf{j}}(\mathrm{r})$ with

$$
\Gamma(r, s, s) \leqslant \theta(g(r))(g(r)-g(s)) .
$$

Then there is $w \in \mathrm{U}$ such that $w \in \bigcap_{\mathrm{j} \in \mathrm{J}} \mathrm{T}_{\mathrm{j}}(w)$, and $\Gamma(w, w, w)=\mathrm{o}$.

Proof. By Theorem 2.15, there exists $w \in U$ such that $\Gamma(w, r, r)>\theta(g(w))(g(w)-g(r))$ for all $r \in U$ with $r \neq w$. Now we show that $w \in \bigcap_{j \in J} \mathrm{~T}_{j}(w)$ and $\Gamma(w, w, w)=0$. According to the assumption, there exists $r(t, j) \in T_{j}(w)$ such that $\Gamma(w, t, t) \leqslant \theta(g(t))(g(w)-g(t(w, j)))$. We show that $t(w, j)=w$ for all $j \in J$. On the contrary, let $\mathrm{t}\left(w, j_{0}\right) \neq w$ for some $j_{0} \in \mathrm{J}$, then

$$
\Gamma(w, t, t) \leqslant \theta(g(w))(g(w)-g(t))<\Gamma(w, t, t),
$$

which is a contradiction. Therefore $w=t(w, j) \in T_{j}(w)$ for all $j \in T$.

Since $\Gamma(w, w, w) \leqslant \theta(g(w))(g(w)-g(w))=0$, we obtain $\Gamma(w, w, w)=0$.

Remark 2.17. We conclude that Theorem 2.16 concludes Theorem 2.15.

On the contrary, for each $r \in U$, there exists $s \in U$ with $s \neq r$ such that

$$
\Gamma(r, s, s) \leqslant \theta(g(r))(g(r)-g(s)) .
$$


Put $\mathrm{T}: \mathrm{U} \longrightarrow 2^{\mathrm{U}} \backslash\{\emptyset\}$ by

$$
\mathrm{T}(\mathrm{r})=\{\mathrm{s} \in \mathrm{U}: \mathrm{s} \neq \mathrm{r}, \Gamma(\mathrm{r}, \mathrm{s}, \mathrm{s}) \leqslant \theta(\mathrm{g}(\mathrm{r}))(\mathrm{g}(\mathrm{r})-\mathrm{fg}(\mathrm{s}))\} .
$$

By Theorem 2.16, $\mathrm{T}$ has a fixed point $w \in \mathrm{U}$, this means, $w \in \mathrm{T}(w)$. This is a contradiction, because $w \notin \mathrm{T}(w)$.

Theorem 2.18 (Nonconvex maximal element theorem for a family of multivalued maps). Assume that $(\mathrm{U}, \mathrm{G})$ is a complete $q-\mathrm{G}-m$ space and $\mathrm{g}: \mathrm{U} \longrightarrow(-\infty, \infty]$ be a proper, bounded below and Lsca function. $\Gamma$ is a $\Gamma$-function on $\mathrm{U} \times \mathrm{U} \times \mathrm{U}$, and $\mathrm{J}$ be any index set. For each $\mathrm{j} \in \mathrm{J}$, let $\mathrm{T}_{\mathrm{j}}: \mathrm{U} \longrightarrow 2^{\mathrm{U}}$ be a multivalued map. Suppose that for each $(\mathrm{r}, \mathrm{j}) \in \mathrm{U} \times \mathrm{J}$ with $\mathrm{T}_{\mathrm{j}}(\mathrm{r}) \neq \emptyset$, there exists $\mathrm{s}=\mathrm{s}(\mathrm{r}, \mathrm{j}) \in \mathrm{U}$ with $\mathrm{s} \neq \mathrm{r}$ such that (2.1) holds. Then there exists $w \in U$ such that $\mathrm{T}_{\mathbf{j}}(\mathrm{W})=\emptyset$ for each $\mathrm{j} \in \mathrm{J}$.

Proof. By Theorem 2.15, there exists $w \in \mathrm{U}$, such that $\Gamma(w, r, r)>\theta(g(w))(g(w)-f(r))$ for all $r \in U$ with $r \neq w$. We prove that $T_{j}(w)=\emptyset$ for each $j \in J$. Indeed, if $T_{j_{0}}(w) \neq \emptyset$, for some $j_{0} \in J$, according to the assumption, there exists $\mathrm{t}=\mathrm{t}\left(w, j_{0}\right) \in \mathrm{U}$ with $\mathrm{t} \neq w$ such that $\theta(w, \mathrm{t}, \mathrm{t}) \leqslant \theta(\mathrm{g}(w))(g(w)-\mathrm{g}(\mathrm{t}))$. Also $\Gamma(w, t, t)>\theta(g(w))(g(w)-g(t))$, which is a contradiction.

Remark 2.19. We conclude that Theorem 2.18 concludes Theorem 2.15.

On the contrary, thus for each $r \in U$, there exists $s \in U$ with $s \neq r$ such that

$$
\Gamma(r, s, s) \leqslant \theta(g(r))(g(r)-g(s)) .
$$

For each $r \in U$, we define $T(r)=\{s \in U: s \neq r,(r, s, s) \leqslant \theta(g(r))(g(r)-g(s))$. Then $T(r) \neq \emptyset$ for all $r \in U$. But by Theorem 2.18, there exists $w \in U$ such that $T(w)=\emptyset$, which is a contradiction.

\section{Nonconvex optimization and minimax theorems}

Theorem 3.1 (Generalized Takahashi's nonconvex minimization theorem). Assume that $(\mathrm{U}, \mathrm{G})$ is a complete $q$-G-m space and $\mathrm{g}: \mathrm{U} \longrightarrow(-\infty, \infty]$ be a proper, bounded below and Lsca function. $\Gamma$ is a $\Gamma$-function on $\mathrm{U} \times \mathrm{U} \times \mathrm{U}$. Suppose that for any $\mathrm{r} \in \mathrm{U}$ with $\mathrm{g}(\mathrm{r})>\inf _{w \in \mathrm{U}} \mathrm{fg}(w)$ there exists $\mathrm{s} \in \mathrm{U}$ with $\mathrm{s} \neq \mathrm{r}$ such that (2.1) holds. Then there exists $w \in \mathrm{U}$ such that $\mathrm{g}(w)=\inf _{\mathrm{t} \in \mathrm{U}} \mathrm{g}(\mathrm{t})$.

Proof. By Theorem 2.15, there exists $w \in U$ such that $\Gamma(w, r, r)>\theta(g(w))(g(w)-g(r))$ for all $r \in U$, $r \neq w$. Now we prove that $g(w)=\inf _{t \in u} g(t)$.

On the contrary, then $g(w)>\inf _{t \in U} g(t)$. According to the assumption, there exists $s=s(w) \in U$, with $s \neq w$ such that $\Gamma(w, s, s) \leqslant \theta(g(w))(g(w)-g(s))$. Then we have $\Gamma(w, s, s) \leqslant \theta(g(w))(g(w)-g(s))<$ $\Gamma(w, s, s)$, which is a contradiction.

Remark 3.2. Using Theorem 3.1, we can conclude Theorem 2.15.

On the contrary, then for each $r \in U$, there exists $s \in U$ with $s \neq r$ such that $\Gamma(r, s, s) \leqslant \theta(g(r))(g(r)-$ $\mathrm{g}(\mathrm{s}))$. By Theorem 3.1, there exists $w \in \mathrm{U}$ such that $\mathrm{g}(w)=\inf _{\mathrm{t} \in \mathrm{U}} \mathrm{g}(\mathrm{t})$. According to the assumption, there exists $z \in \mathrm{U}$ with $z \neq \mathrm{r}$, such that $\Gamma(w, z, z) \leqslant \theta(g(w))(g(w)-g(z)) \leqslant 0$. Then $\Gamma(w, z, z)=0$ and $g(w)=g(z)=\inf _{t \in U} g(t)$. There exists $t \in U$ with $t \neq z$ such that $\Gamma(z, t, t) \leqslant \theta(g(z))(g(z)-g(t)) \leqslant 0$. Then we have $\Gamma(z, t, t)=0$ and $g(w)=g(z)=g(t)=\inf _{r \in u} g(r)$. Since $\Gamma(w, t, t) \leqslant \Gamma(w, z, z)+\Gamma(z, t, t)$, then $\Gamma(w, t, t)=0$. For $\epsilon>0$ we have $\Gamma(w, z, z)=0<\delta, \Gamma(z, t, t)=0<\delta$ then $\mathrm{G}(w, t, t)<\epsilon$, that is, $w=t$. Also for $\epsilon>0$ we have $\Gamma(z, w, w)=0<\delta, \Gamma(w, t, t)=0<\delta$, then $\mathrm{G}(z, t, t)<\epsilon$ that is, $z=t$, which is a contradiction.

Theorem 3.3 (Nonconvex minimax theorem). Assume that $(\mathrm{U}, \mathrm{G})$ is a complete $q-\mathrm{G}-m$ space and $\Gamma$ is a $\Gamma$ function on $\mathrm{U} \times \mathrm{U} \times \mathrm{U}$. Let $\mathrm{F}: \mathrm{U} \times \mathrm{U} \rightarrow(-\infty, \infty]$ be a proper lsca and bounded below function in the first argument. Suppose that for each $\mathrm{r} \in \mathrm{U}$ with $\left\{x \in \mathrm{U}: \mathrm{F}(\mathrm{r}, \mathrm{x})>\inf _{\mathrm{a} \in \mathrm{U}} \mathrm{F}(\mathrm{a}, \mathrm{x})\right\} \neq \emptyset$, there exists $\mathrm{s}=\mathrm{s}(\mathrm{r}) \in \mathrm{U}$ with $\mathrm{s} \neq \mathrm{r}$ such that

$$
\Gamma(r, s, s) \leqslant \theta(F(r, w))(F(r, w)-F(s, w))
$$

for all $w \in\left\{x \in U: F(r, x)>\inf _{a \in U} F(a, x)\right\}$. Then $\inf _{r \in U} \sup _{s \in U} F(u, s)=\sup _{s \in U} \inf _{r \in U} F(r, s)$. 
Proof. By Theorem 3.1, for every $s \in U$, there exists $r(s) \in U$ such that $F(r(s), s)=\inf _{r \in U} F(r, s)$. Then $\sup _{s \in U} F(r(s), s)=\sup _{s \in U} \inf _{r \in U} F(r, s)$.

By displacement of $r(s)$ with an arbitrary $r \in U$ and then getting inf, we obtain $\inf _{r \in U} \sup _{s \in U} F(r, s)=$ $\sup _{s \in U} \inf _{r \in U} F(r, s)$.

Theorem 3.4 (Nonconvex equilibrium theorem). Assume that $(\mathrm{U}, \mathrm{G})$ is a complete $q-\mathrm{G}-m$ space and $\Gamma$ is a $\Gamma$-function on $\mathrm{U} \times \mathrm{U} \times \mathrm{U}$. Let $\mathrm{F}$ and $\theta$ be the same as in Theorem 3.3. Let, for each $\mathrm{r} \in \mathrm{U}$ with $\{\mathrm{x} \in \mathrm{U}: \mathrm{F}(\mathrm{r}, \mathrm{x})<$ $0\} \neq \emptyset$, there exists $\mathrm{s}=\mathrm{s}(\mathrm{r}) \in \mathrm{U}$ with $\mathrm{s} \neq \mathrm{r}$ such that (3.1) holds for all $\mathrm{t} \in \mathrm{U}$. Then there exists $\mathrm{y} \in \mathrm{U}$ such that $\mathrm{F}(\mathrm{y}, \mathrm{s}) \geqslant 0$ for all $\mathrm{s} \in \mathrm{U}$.

Proof. From Theorem 2.15 for each $t \in U$, there exists $y(t) \in U$ such that $\Gamma(y(t), r, r)>\theta(F(y(t), t))(F(y(t)$, $t)-F(r, t))$ for all $r \in U$ with $r \neq y(t)$. We show that there exists $y \in U$ such that $F(y, s) \geqslant 0$ for all $s \in U$. On the contrary, for each $r \in U$ there exists $s \in U$ such that $F(r, s)<0$. Then for each $r \in U$, $\{x \in U: F(r, x)<0\} \neq \emptyset$. According to the assumption, there exists $s=s(y(t)), y \neq y(t)$ such that $\Gamma(y(t), s, s) \leqslant \theta(F(y(t), t))(F(y(t), t)-F(s, t))$, which is a contradiction.

Example 3.5. Let $\mathrm{U}=[0,1]$ and $\mathrm{G}(\mathrm{r}, \mathrm{s}, \mathrm{t})=\max \{|r-s|,|r-t|,|s-t|\}$. Then $(X, \mathrm{G})$ is a complete $\mathrm{q}-\mathrm{G}$ $m$ space. Suppose that $a, b$ be positive real numbers with $a \geqslant b$. Suppose $H: U \times U \longrightarrow R$ with $H(r, s)=\frac{a}{2} r-\frac{b}{3} s$. Therefore, function $r \longrightarrow H(r, s)$ is proper, lower semicontinuous and bounded below, and $H(1, s) \geqslant 0$ for every $s \in U$. Also $H(r, s) \geqslant 0$ for every $r \in\left[\frac{b}{a}, 1\right]$ and for every $s \in U$. In fact, for every $r \in\left[0, \frac{b}{a}\right], H(r, s)=a r-b s<0$ when $s \in\left[\frac{a}{b} r, 1\right]$. Then set $\{x \in U: H(r, x)<0\} \neq \emptyset$ for every $r \in\left[0, \frac{b}{a}\right]$. Let $r, s \in U, r \geqslant s$, we have $r-s=\frac{2}{a}\left\{\left(\frac{a}{2} r-\frac{b}{3} x\right)-\left(\frac{a}{2} s-\frac{b}{3} x\right)\right\}$ for every $x \in U$. Let $\theta:[0, \infty) \longrightarrow[0, \infty)$ with $\theta(t)=\frac{2}{a}$ be defined. Therefore $G(r, s, s) \leqslant \theta(H(r, x))(H(r, x)-H(s, x))$ for every $r \geqslant s$, and $r, s, x \in U$. By Theorem 3.4 there exists $y \in U$ such that $H(y, s) \geqslant 0$ for every $s \in U$.

\section{Applications}

Definition 4.1. Let $(\mathrm{U}, \mathrm{G})$ be a $\mathrm{q}-\mathrm{G}-\mathrm{m}$ space and $\mathrm{a}, \mathrm{b} \in \mathrm{U}$. Suppose that $\lambda: \mathrm{U} \rightarrow(0, \infty)$ be a function and $\Gamma$ be a $\Gamma$-function on $U$. Define

$$
\Gamma_{\epsilon}(a, b, \lambda)=\{r \in U: \epsilon \Gamma(a, r, r) \leqslant \lambda(a)(\Gamma(b, a, a)-\Gamma(b, r, r))\}
$$

such that $\epsilon \in(0, \infty)$ and $a, b \in U$.

Lemma 4.2. Assume that $(\mathrm{U}, \mathrm{G})$ is a complete $q-\mathrm{G}-m$ space and $\mathrm{g}: \mathrm{U} \longrightarrow(-\infty, \infty]$ be a proper, bounded below and Lsca function and $\Gamma$ is a $\Gamma$-function on $\mathrm{U} \times \mathrm{U} \times \mathrm{U}$. Let $\epsilon>0$. Suppose that there exists $\mathrm{x} \in \mathrm{U}$ such that $\mathrm{g}(\mathrm{x})<\infty$ and $\Gamma(x, x, x)=0$. Then there exists $\mathrm{t} \in \mathrm{U}$ such that

(i) $\epsilon \Gamma(x, t, t) \leqslant \theta(g(x))(g(x)-g(t))$;

(ii) $\Gamma(\mathrm{t}, \mathrm{r}, \mathrm{r})>\theta(\mathrm{g}(\mathrm{t}))(\mathrm{g}(\mathrm{t})-\mathrm{g}(\mathrm{r}))$ for all $\mathrm{r} \in \mathrm{U}$ with $\mathrm{r} \neq \mathrm{t}$.

Proof. Let $x \in \mathrm{U}, \mathrm{g}(\mathrm{x})<+\infty$ and $\Gamma(x, x, x)=0$. Put

$$
S=\{r \in U: \epsilon \Gamma(x, r, r) \leqslant \theta(g(x))(g(x)-g(r)) .
$$

Therefore $(S, G)$ is a nonempty complete $\mathrm{q}-\mathrm{G}-\mathrm{m}$ space. By Theorem 2.15 , there exists $\mathrm{t} \in \mathrm{S}$ such that $\epsilon \Gamma(t, r, r)>\theta(g(t))(g(t)-g(r))$ for all $r \in S$ with $r \neq t$. For any $r \in U \backslash S$, since $\epsilon[\Gamma(x, t, t)+\Gamma(t, r, r)] \geqslant$ $\epsilon \Gamma(x, r, r)>\theta(g(x))(g(x)-g(r)) \geqslant \epsilon \Gamma(x, t, t)+\theta(g(t))(g(t)-g(r))$, therefore $\epsilon \Gamma(t, r, r)>\theta(g(t))(g(t)-$ $g(r))$ for all $r \in U \backslash S$. Then $\epsilon \Gamma(t, r, r)>\theta(g(t))(g(t)-g(t))$ for all $r \in U$ with $r \neq t$.

Theorem 4.3 (Generalized flower petal theorem). Suppose that $P$ be a proper complete subset of a $q-G-m$ space $U$ and $a \in P$. Let $\Gamma$ be $a \Gamma$-function on $U$ with $\Gamma(a, a, a)=0$. Let $b \in U \backslash P, \Gamma(b, P, P)=\inf _{r \in P} \Gamma(b, r, r) \geqslant u$ and $\Gamma(b, a, a)=s>0$ and there exists a function $\lambda$ from $U$ into $(0, \infty)$ satisfying $\lambda(r)=\theta(\Gamma(b, r, r))$ for some nondecreasing function $\theta$ from $(-\infty, \infty]$ into $(0, \infty)$. Then for each $\epsilon>0$, there exists $t \in P \cap \Gamma_{\epsilon}(a, b, \lambda)$ such that $\Gamma_{\epsilon}(t, b, \lambda) \bigcap(P \backslash\{t\})=\emptyset$ and $(a, t, t) \leqslant \epsilon^{-1} \lambda(a)(s-r)$.

Proof. $(P, G)$ is a complete $q-G-m$ space. Consider $g: P \longrightarrow(-\infty, \infty], g(r)=\Gamma(b, r, r)$. Since $g(a)=$ 
$\Gamma(b, a, a)=s<\infty$ and $\Gamma(b, P, P)=\inf _{r \in P} \Gamma(b, r, r) \geqslant u$ then $g$ is a proper lower semicontinuous and bounded below function. By Lemma 4.2, there exists $t \in P$ such that

(i) $\epsilon \Gamma(a, t, t) \leqslant \lambda(a)(g(a)-g(t))$;

(ii) $\epsilon \Gamma(t, r, r)>\lambda(t)(g(t)-g(t))$ for all $r \in P$ with $r \neq t$.

Applying (i), we have $t \in P \cap \Gamma_{\epsilon}(a, b, \lambda)$. Also, applying (i) again, we have $\Gamma(a, t, t) \leqslant \epsilon^{-1} \lambda(a)(\Gamma(b, a, a)-$ $\Gamma(b, t, t)) \leqslant \epsilon^{-1} \lambda(a)(s-r)$. By (ii), we obtain $\epsilon(t, r, r)>\lambda(t)(\Gamma(b, t, t)-\Gamma(b, r, r))$ for all $r \in P$ with $r \neq t$. Therefore $u \notin \Gamma_{\epsilon}(t, b, \lambda)$ for all $r \in P \backslash\{t\}$ or $\Gamma_{\epsilon}(t, b, \lambda) \bigcap(P \backslash\{t\})=\emptyset$.

\section{References}

[1] M. Amemiya, W. Takahashi, Fixed point theorems for fuzzy mappings in complete metric spaces, Fuzzy Sets and system, 125 (2002), 253-260. 1

[2] J.-P. Aubin, J. Siegel, Fixed points and stationary points of dissipative multivalued maps, Proc. Amer. Math. Soc., 78 (1980), 391-398. 1

[3] J. S. Bae, Fixed point theorems for weakly contractive multivalued maps, J. Math. Anal. Appl., 284 (2003), 690-697.

[4] J. S. Bae, E. W. Cho, S. H. Yeom, A generalization of the Caristi-Kirk fixed point theorem and its application to mapping theorems, J. Korean Math. Soc., 31 (1994), 29-48. 1

[5] J. Caristi, Fixed point theorems for mappings satisfying inwardness conditions, Trans. Amer. Math. Soc., 215 (1976), 241-251.

[6] Y. Chen, Y. J. Cho, L. Yang, Note on the results with lower semi-continuity, Bull Korean Math. Soc., 39 (2002), $535-541$.

[7] P. Z. Daffer, H. Kaneko, W. Li, Variational principle and fixed points, in: Set Valued Mappings With Applications in Nonlinear Analysis, 2002 (2002), 129-136. 1

[8] S. Dancs, M. Hegedüs, P. Medvegyev, A general ordering and fixed point principle in complete metric spaces, Acta. Sci. Math. (Szeged), 46 (1983), 381-388. 1

[9] I. Ekeland, Nonconvex minimization problems, Bull. Amer. Math. Soc., 1 (1979), 443-474. 2.1

[10] I. Ekeland, On the variational principle, J. Math. Anal. Appl., 47 (1974), 324-353. 1

[11] I. Ekeland, Remarques sur les problémes variationnels, C. R. Acad. Sci. Paris Sér. A-B, 275 (1972), 1057-1059. 1

[12] L. Gajek, D. Zagrodny, Geometric variational principle, Dissertationes Math. (Rozprawy Mat.), 340 (1995), 55-71. 1

[13] A. Hamel, Remarks to an equivalent formulation of Ekelands variational principle, Optimization, 31 (1994), $233-238$.

[14] A. Hamel, A. Löhne, A minimal point theorem in uniform spaces, in: Nonlinear analysis and applications: to V. Lakshmikantham on his 80th birthday, 1, 2 (2003), 557-593.

[15] D. H. Hyers, G. Isac, T. M. Rassias, Topics in Nonlinear Analysis and Applications, World Scientific Publishing Co., River Edge, (1997).

[16] O. Kada, T. Suzuki, W. Takahashi, Nonconvex minimization theorems and fixed point theorems in complete metric spaces, Math. Japon., 44 (1996), 381-391. 1

[17] Y. Kijima, On a minimization theorem, in: Nonlinear Analysis and Convex Analysis (Japanese), 1995 (1995), $59-62$. 1

[18] N. Mizoguchi, W. Takahashi, Fixed point theorems for multivalued mappings on complete metric spaces, J. Math. Anal. Appl., 141 (1989), 177-188. 1

[19] Z. Mustafa, B. Sims, A new approach to generalized metric spaces, J. Nonlinear Convex Anal., 7 (2006) 289-392. 2.2, $2.3,2.4$

[20] J.-P. Penot, The drop theorem, the petal theorem and Ekelands variational principle, Nonlinear Anal., 10 (1986), 813-822. 1

[21] R. Saadati, S. M. Vaezpoura, P. Vetro , B. E. Rhoades, Fixed point theorems in generalized partially ordered G-metric spaces, Math. Comput. Modelling, 52 (2010), 797-801. 2.8, 2.11

[22] N. Shioji, T. Suzuki, W. Takahashi, Contractive mappings, Kannan mappings and metric completeness, Proc. Amer. Math. Soc., 126 (1998), 3117-3124. 1

[23] T. Suzuki, Generalized distance and existence theorems in complete metric spaces, J. Math. Anal. Appl., 253 (2001), 440-458. 1

[24] T. Suzuki, On DowningKirks theorem, J. Math. Anal. Appl., 286 (2003), 453-458.

[25] T. Suzuki, Generalized Caristis fixed point theorems by Bae and others, J. Math. Anal. Appl., 302 (2005), 502-508.

[26] T. Suzuki, W. Takahashi, Fixed point theorems and characterizations of metric completeness, Topol. Methods Nonlinear Anal., 8 (1996), 371-382.

[27] W. Takahashi, Existence theorems generalizing fixed point theorems for multivalued mappings, in: Fixed Point Theory and Applications, 1991 (1991), 397-406.

[28] W. Takahashi, Nonlinear Functional Analysis, Yokohama Publishers, Yokohama, (2000).

[29] D. Tataru, Viscosity solutions of HamiltonJacobi equations with unbounded nonlinear terms, J. Math. Anal. Appl., 163 (1992), 345-392.

[30] C.-K. Zhong, On Ekelands variational principle and a minimax theorem, J. Math. Anal. Appl., 205 (1997), 239-250. 1 